American Journal of Applied Sciences 5 (5): 490-495, 2008

ISSN 1546-9239

(C) 2008 Science Publications

\title{
A Novel Approach for Testing Stability of 1-D Recursive Digital Filters Based on Lagrange Multipliers
}

\author{
${ }^{1}$ K.R.Santhi, ${ }^{1}$ N.Gangatharan and ${ }^{2}$ M.Ponnavaikko \\ ${ }^{1}$ Faculty of Engineering, Kigali Institute of Science and Technology (KIST), Kigali, B.P.3900, Rwanda \\ and ${ }^{2}$ Directorate of Research and Virtual Education, SRM University, SRM Nagar, Kattangulathur, \\ 603203, TamilNadu, Kanchepuram Dt, India
}

\begin{abstract}
Digital filters have found their way into many products from every day consumer items such as mobile phones to advanced maritime and military communications and avionics systems. Design of digital filters faces two fundamental problems, their stability and synthesis. Recursive filters have more stability problems than nonrecursive filters. Stability of a filter can be determined by the location of the zero valued region of the denominator polynomial of its transfer function. Stability of recursive filters has been studied by many researchers for the past three decades. Several theorems on stability testing and stabilizing recursive digital filters have been already proposed. We present a new approach to test the stability problem of the one-dimensional (1-D) recursive digital filters using Lagrange Multipliers. This method not only tests the stability of recursive digital filters, but also provides the stable version of the filter's transfer function if found to be unstable.
\end{abstract}

Keywords: Digital Filters, Lagrange Multiplier, Autocorrelation, Stability, Least Squares Inverse

\section{INTRODUCTION}

Digital filters are used in many digital signal processing (DSP) fields, but the recursive version of which is difficult to design due to stability problem. Several filtering applications, such as in image processing or in communications, require a linear-phase characteristic. This can be easily achieved with finiteimpulse response (FIR) filters, also known as nonrecursive filters. However, the resulting FIR filter order is sometimes exceedingly high, producing an unacceptable delay and computational cost. The alternative is to use infinite-impulse response (IIR) filters known also as recursive filters with approximate linear-phase. For a given filter specifications, the required order for a recursive filter is 5 to 10 times lower than that of a nonrecursive filter ${ }^{[1]}$. In recursive filters, the poles of the transfer function (equivalently, the zeros of the denominator polynomial of the filter's transfer function) can be placed anywhere inside the unit circle in the z-plane. As a consequence of this degree of freedom, high selectivity filters can easily be designed with lower-order transfer functions. In nonrecursive filters, on the other hand, with poles fixed at origin, high selectivity can be achieved only by using a relatively higher order transfer functions ${ }^{[2]}$. A general desire in any filter design is that the number of operations needed to compute the filter response is as low as possible. The order of a filter is more or less proportional to the number of operations. Recursive filter is preferable, primarily because of its lower order. So its implementation involves fewer parameters, requires less memory and has lower computational complexity than the nonrecursive counterpart ${ }^{[3]}$. This gives more flexibility and power to implement high selectivity recursive filters using smaller number of coefficients meeting a particular magnitude specification, but results in stability problems in both design and implementation.

A recursive digital filter is defined to be BoundedInput-Bounded-Output (BIBO) stable if every bounded input results in a bounded output. Stability is often a desirable constraint to impose since an unstable system can generate an unbounded output, which can cause system overload and other difficulties ${ }^{[4,6]}$. The stability testing and stabilization of 1-D recursive digital filters has been studied by several researchers ${ }^{[2,5]}$. In this paper a new method of stability testing and stabilizing 1-D recursive digital filters based on Lagrange multipliers is obtained.

Corresponding Author: $\quad$ K.R.Santhi, Department of Computer Engineering and Information Technology, Faculty of Engineering, Kigali Institute of Science and Technology (KIST), PO Box 3900, Rwanda 


\section{1-D RECURSIVE DIGITAL FILTER STABILITY THEOREMS AND STABILIZATION METHODS}

In this section, the various stability theorems and stabilization methods on 1-D recursive digital filters are reviewed and compared. Recursive filters are conveniently described in terms of 1-D z-transforms. It is assumed throughout this section that z-transform is defined with negative powers of $z$. Under this assumption, stability implies that all poles of the filter's transfer function must be within the unit circle.

Consider the transfer function $H(z)$ of a certain 1$D$ recursive digital filter given by

$$
H(z)=\frac{1}{A(z)}=\frac{1}{\sum_{k=0}^{M} a_{k} z^{-k}}
$$

One method of testing the stability of the filter described by the transfer function as in (1) is to explicitly determine the zeros of $A(z)$ by a root finding method $^{[3,6]}$ and check if all the zeros are inside the unit circle of the complex z-plane. If so, the system described by the transfer function (1) is stable else unstable. This method is conceptually very simple, and is computationally efficient for low values of $M$ where, $M$ is the order of the denominator polynomial of (1). For values of $M$ up to $M=4$, closed-form expressions can be used to determine the zeros. Even if the closedform expressions are not available, the zeros determined in one test can be used as initial estimates for the zeros in the subsequent test. This is because the zeros obtained in the 1-D stability tests typically do not vary much from one test to the other. This method has been reported ${ }^{[2]}$ to be quite useful in practice for $M=8$. The explicit evaluation of the zeros using this method provides us considerable insight into exactly how stable a stable filter is. If none of the zeros determined is very close to the unit circle, the filter is said to be stable ${ }^{[3,6]}$.

The second method of testing the stability of the filter described by the transfer function (1) is to use Marden-Jury Test ${ }^{[6]}$ which is proven to be computationally efficient. This method has been reported to be computationally efficient and reliable for $M$ up to 20 that cover most recursive filters considered in practice ${ }^{[2]}$. However, the disadvantage being it does not tell us how stable a filter is. The method can be used in determining the number of zeros inside the unit circle, but cannot be used explicitly to determine the locations of the zeros.
The third method of testing the filter for stability is to exploit the Argument Approach ${ }^{[2]}$. Consider the net change in the argument of $A(z)$ in (1) as we follow the unit circle contour given by $z=e^{j \omega}$ from $\omega=0$ to $\omega=2 \pi$ in a counterclockwise direction. Denoting the net argument change by $\Delta \theta_{A}(\omega: 0,2 \pi)$, the argument approach states that

$\Delta \theta_{A}(\omega: 0,2 \pi)=2 \pi\left(N_{z}-M\right)$

where, $N_{Z}$ is the number of zeros inside the unit circle. When all roots are inside the unit circle, $N_{Z}=M$ and therefore,

$\Delta \theta_{A}(\omega: 0,2 \pi)=0$

From (3) it is clear that, to test the stability is to check if the net phase change is zero. The net phase change can be determined by unwrapping the phase. If the unwrapped phase is continuous and the unwrapped phases at $\omega=0$ and $\omega=2 \pi$ are identical, the system is stable, else it is unstable. This method has been reported to be reliable and computationally efficient in comparison to other methods for $M>20^{[2]}$. It is also noted that the phase unwrapping is quite difficult when some roots are very close to the unit circle. The unwrapped phase has some qualitative features that can be related to the degree of system stability. For a stable filter, rapid change in the unwrapped phase typically occurs when some zeros are close to the unit circle.

In 1-D systems there are two standard approaches to design recursive filters. One approach is to design the filter from an analog system function and the other is to design directly in the discrete domain. The direct design method is further classified into spatial domain and frequency domain methods. In the spatial domain method, the filters are designed by using an error criterion in the spatial domain, conversely in the frequency domain method the filters are designed using an error criterion in the frequency domain. The filters designed by both spatial domain and frequency domain techniques can still be unstable.

There are few methods by which the 1-D recursive digital filter can be stabilized. The popular methods are Least Squares Inverse (LSI) ${ }^{[5,7]}$ and Discrete Hilbert Transform (DHT) methods ${ }^{[8]}$. It has been shown ${ }^{[8]}$ that DHT method of stabilization will yield stable polynomials if the original polynomial does not have zeros on the unit circle. Likewise, it is well known ${ }^{[5,9-}$ ${ }^{11]}$ that the LSI of a 1-D polynomial that does not have zeros on the unit circle is always stable. This fact can be utilized for the effective design of stable 1-D recursive digital filters. In the process of obtaining the stable LSI 
polynomial corresponding to an unstable polynomial, since the minimum error $[5,10,14]$ is given by

$E_{\text {min }}=1-a_{0} b_{0}$

the value of $b_{0}$ will always turn out to be always the highest value. In this, $a_{0}$ and $b_{0}$ are the constant terms of the original and LSI polynomials respectively. It is apparent that the stable LSI polynomial will have the highest value for its constant term. We use this concept of maximizing the constant term of a polynomial to test the stability or otherwise of a polynomial in the following sections.

\section{SOME IMPORTANT PRELIMINARIES AND CONCEPTS}

Unlike the conventional method of defining $\mathrm{z}$ transform of a discrete signal ${ }^{[6]}$, it is assumed hereafter that positive powers of $\mathrm{z}$ are used in the definition of $\mathrm{z}$ transform ${ }^{[12,13]}$. With this assumption, we have the following Theorem ${ }^{[2]}$.

\section{Theorem 1:}

(i) The 1-D polynomial $A(z)$ is stable if and only if $A(z) \neq 0$, for all $|z| \leq 1$

(ii) A stable $A(z)$ is said to be marginally stable if $A(z)=0$, for some $|z|=1$

We also have the following definition on autocorrelation coefficients [10].

\section{Definition:}

If $A(z)$ is an $M^{\text {th }}$ degree polynomial as in (5) the coefficients $\gamma_{s}$ 's of $A(z) A\left(z^{-1}\right)$ written as

$$
A(z) A\left(z^{-1}\right)=\gamma_{0}+\gamma_{1}\left(z+z^{-1}\right)+\gamma_{2}\left(z^{2}+z^{-2}\right)+\ldots . .+
$$$$
\gamma_{M}\left(z^{M}+z^{-M}\right)
$$

are called the autocorrelation coefficients of $A(z)$. The coefficients are $(M+1)$ in number. For a real polynomial $A(z), \gamma_{s}$ 's are real.

Consider the transfer function of a certain recursive digital filter such that $H(z)=\frac{1}{A(z)}$ where,

$$
A(z)=\sum_{k=0}^{M} a_{k} z^{k}
$$

be of degree $M$. It has $(M+1)$ autocorrelation functions $\gamma_{s}$ 's as given below:
$\sum_{r=0}^{M} a_{r} a_{r+s}=\gamma_{s}, \quad s=0,1,2, \ldots \ldots . M$

We would like to point out that for any given 1-D polynomial $A(z)$ of order $\mathrm{M}$, there are $2^{M}$ number of 1-D polynomials in total (without counting the negatives of these polynomials) which will have the same autocorrelation coefficients $\gamma_{s}$ 's as that of $A(z)$ as shown in later sections. Out of these $2^{M}$ number of 1-D polynomials, which are said to form a family, only one polynomial will lead to a stable system. It has been shown that the autocorrelation functions or coefficients are related to magnitude square of system functions ${ }^{[15]}$. Therefore it is obvious all the $2^{M}$ number of polynomials will have the same magnitude response. Out of this $2^{M}$ number of polynomials in total only one polynomial will be stable for which the constant term will have the highest value as discussed in the foregoing section. The remaining polynomials although provide the same magnitude response, may lead to unstable system. We now state this in the form of a theorem.

\section{Theorem 2:}

For any 1-D polynomial $A(z)$ of degree $\mathrm{M}$, there are $2^{M}$ distinct 1-D polynomials with same autocorrelation function that form a family. Only one 1-D polynomial among the family whose constant term is the highest leads to a stable system.

Based on Theorem 3.2 we use the Lagrange multipliers to find out the stable polynomial. The proof of the above theorem is shown in the following section.

\section{STABILITY TESTING AND STABILIZATION BASED ON LAGRANGE MULTIPLIERS}

In this section, a new method of testing the stability and stabilizing 1-D recursive digital filters based on Lagrange multipliers is explained. This method aims to maximize the constant term of the 1-D polynomial and a decision regarding its stability can be made depending upon whether the constant term of the 1-D polyngmial is maximizable or not. To test the stability of a system described by the transfer function (1) whose denominator polynomial is $A(z)$, we use Lagrange multipliers. 
Let the stable version of $A(z)$ be $A^{\prime}(z)$ whose coefficients are denoted by $a_{r}^{\prime}$. To show that the given polynomial $A(z)$ is stable, we should maximize the constant term $a_{0}^{\prime}$ or $a_{0}^{\prime 2}$ of $A^{\prime}(z)$ by using Lagrange multipliers.

Let ' $f$ ' be the function to be maximized

$f=a_{0}^{\prime 2}$

satisfying the constraints $g_{i}$, given as

$g_{i}=\sum_{r=0}^{M} a_{r}^{\prime} a_{r+s}^{\prime}-\gamma_{s}=0, \quad s=0,1,2, \ldots \ldots . M$

where, $\gamma_{s}=\sum_{r=0}^{M} a_{r} a_{r+s} \quad s=0,1,2, \ldots \ldots . . M$

that is,

$g_{i}=0, \quad i=0,1,2, \ldots \ldots \ldots \ldots$

The above constraints are framed due to the fact that the constant term $a_{0}$ of one of the polynomials from the family will be equal to the constant term $a_{0}^{\prime}$ of the assumed stable version of the polynomial $A^{\prime}(z)$ if $A(z)$ is the stable polynomial among the family. Here, $\gamma_{s}$ represents the autocorrelation coefficients as in (6) of the original polynomial $A(z)$ and $\gamma_{s}^{\prime}=\sum_{r=0}^{M} a_{r}^{\prime} a_{r+s}^{\prime}$ represents the autocorrelation coefficients of the stable version $A^{\prime}(z)$.

Let us now form the Lagrange Function $L\left(a_{0}^{\prime}, \lambda_{i}\right)$ such that

$$
L\left(a_{0}^{\prime}, \lambda_{i}\right)=f+\sum_{i=0}^{M} \lambda_{i} g_{i}
$$

where $\lambda_{i}$ 's are the Lagrange multipliers.

The necessary and sufficient conditions for the function ' $f$ ' to be global maximum and hence ' $a$ ' ' to be the greatest in magnitude are given using Lagrange multiplier method as follows ${ }^{[13]}$.

$$
\frac{\partial f}{\partial a_{0}^{\prime}}+\sum_{i=0}^{M} \lambda_{i} \frac{\partial g_{i}}{\partial a_{0}^{\prime}}=0
$$

and

$$
\lambda_{i}>0, i=0,1,2, \ldots, M
$$

For a given polynomial $A(z)$, all the coefficients $a_{r}$ 's are known and hence $\gamma_{s}$ 's are fixed.

If we want to test whether $A(z)$ will lead to a stable system or not, we have to do the following:

Obtain the equation

$\frac{\partial L\left(a_{0}^{\prime}, \lambda_{i}\right)}{\partial a_{0}^{\prime}}=0$

Using (10) in (13) we get,

$\frac{\partial L\left(a_{0}^{\prime}, \lambda_{i}\right)}{\partial a_{0}^{\prime}}=2 a_{0}^{\prime}+2 a_{0}^{\prime} \lambda_{0}+a_{1}^{\prime} \lambda_{1}+a_{2}^{\prime} \lambda_{2}+$
$\ldots . .+a_{M}^{\prime} \lambda_{M}=0$

Simplifying (14), we get,

$a_{0}^{\prime}=\frac{-\left(a_{1}^{\prime} \lambda_{1}+a_{2}^{\prime} \lambda_{2}+\ldots+a_{M}^{\prime} \lambda_{M}\right)}{2\left(1+\lambda_{0}\right)}$

The constraint equations in (8) can be expanded as follows:

$a_{0}^{\prime 2}+a_{1}^{\prime 2}+a_{2}^{\prime 2}+\ldots .+a_{M}^{\prime 2}=\gamma_{0}$
$a_{0}^{\prime} a_{1}^{\prime}+a_{1}^{\prime} a_{2}^{\prime}+\ldots \ldots . .+a_{M-1}^{\prime} a_{M}^{\prime}=\gamma_{1}$

$a_{0}^{\prime} a_{M}^{\prime}=\gamma_{M}$

Substitute the value of $a_{0}^{\prime}$ which was obtained from (15) in the expanded constraint equations (16) above.

This will result in $(\mathrm{M}+1)$ equations involving $(\mathrm{M}+1)$ number of $\lambda_{i}$ 's and $\mathrm{M}$ number of $a_{i}^{\prime}$ 's as unknowns.

$\left[\frac{-\left(a_{1}^{\prime} \lambda_{1}+a_{2}^{\prime} \lambda_{2}+\ldots+a_{M}^{\prime} \lambda_{M}\right)}{2\left(1+\lambda_{0}\right)}\right]^{2}+a_{1}^{\prime 2}+a_{2}^{\prime 2}+\ldots .+a_{M}^{\prime 2}=\gamma_{0}$ $\left[\frac{-\left(a_{1}^{\prime} \lambda_{1}+a_{2}^{\prime} \lambda_{2}+\ldots+a_{M}^{\prime} \lambda_{M}\right)}{2\left(1+\lambda_{0}\right)}\right] a_{1}^{\prime}+a_{1}^{\prime} a_{2}^{\prime}+\ldots \ldots . .+a_{M-1}^{\prime} a_{M}^{\prime}=\gamma_{1}$

$\left[\frac{-\left(a_{1}^{\prime} \lambda_{1}+a_{2}^{\prime} \lambda_{2}+\ldots+a_{M}^{\prime} \lambda_{M}\right)}{2\left(1+\lambda_{0}\right)}\right] a_{M}^{\prime}=\gamma_{M}$

Solve (17) for $a_{i}^{\prime}$ 's and $\lambda_{i}$ 's. In this process all $\lambda_{i}$ 's should turn out to be positive and $a_{i}^{\prime}$ 's real satisfying (11) and (12). The values of $a_{i}^{\prime}$ and $\lambda_{i}$ 's thus obtained from (17) are used to get the maximum value of $a_{0}^{\prime}$ by 
using (15). If the maximum value of $a_{0}^{\prime}$ is the same as $a_{0}$ of the given polynomial $A(z)$, then we can conclude that $A(z)$ will lead to a stable system.

\section{NUMERICAL RESULTS}

In this section, we first show that for any given 1-D polynomial $A(z)$, there are $2^{M}$ number of 1-D polynomials in total which will have the same autocorrelation coefficients $\gamma_{s}$ 's as that of $A(z)$.

Consider a 1-D polynomial of second degree $(\mathrm{M}=2), A(z)=2 z^{2}+7 z+6$, which is of the form $A(z)=a_{2} z^{2}+a_{1} z+a_{0}$. There will be 4 polynomials in total in the family of $A(z)$.

The autocorrelation coefficients of $A(z)$ are as follows:

$\gamma_{0}=a_{0}{ }^{2}+a_{1}{ }^{2}+a_{2}{ }^{2}=6^{2}+7^{2}+2^{2}=89$

$\gamma_{1}=a_{0} a_{1}+a_{1} a_{2}=(6 \times 7)+(7 \times 2)=56$

$\gamma_{2}=a_{0} a_{2}=6 \times 2=12$

There are three other polynomials without counting the negatives of the polynomials in the family for the given $A(z)$. They are as follows:

$$
\begin{aligned}
& A_{1}(z)=3 z^{2}+8 z+4 \\
& A_{2}(z)=4 z^{2}+8 z+3 \\
& A_{3}(z)=6 z^{2}+7 z+3
\end{aligned}
$$

It can be found that all these three polynomials will also have the same autocorrelation coefficients as that of $A(z)$, i.e. 89, 56 and 12. As discussed in previous section, the polynomials which have the same autocorrelation coefficients will also have the same magnitude response and among this only one polynomial will be stable for which the constant term is the highest (magnitude wise). Now we have to show that the polynomial with the highest value for its constant term is stable. It is palpable from our discussion in section IV that the Lagrange multiplier method maximizes the constant term of the given polynomial and hence in the process of maximization if $a_{0}^{\prime}$ turns out to be equal to $a_{0}$, then one can conclude that the given polynomial is stable or not.

Consider again the same 1-D polynomial of second degree

$$
A(z)=2 z^{2}+7 z+6
$$

where $a_{0}=6, a_{1}=7, a_{2}=2$.

Let us now check the stability of this polynomial.
Let $A^{\prime}(z)=a_{2}^{\prime} z^{2}+a_{1}^{\prime} z+a_{0}^{\prime}$

represent the stable version of the given polynomial $A(z)$ having the same autocorrelation coefficients as $A(z)$. The autocorrelation constraint equations of $A^{\prime}(z)$ using (8) are as follows.

$a_{0}^{\prime 2}+a_{1}^{\prime 2}+a_{2}^{\prime 2}=89$

$a_{0}^{\prime} a_{1}^{\prime}+a_{1}^{\prime} a_{2}^{\prime}=56$

$a_{0}^{\prime} a_{2}^{\prime}=12$

By using (15) in (20), we get,

$$
\begin{aligned}
& \frac{\left(a_{1}^{\prime} \lambda_{1}+a_{2}^{\prime} \lambda_{2}\right)^{2}}{4\left(1+\lambda_{0}\right)^{2}}+a_{1}^{\prime 2}+a_{2}^{\prime 2}=89 \\
& \frac{-\left(a_{1}^{\prime} \lambda_{1}+a_{2}^{\prime} \lambda_{2}\right) a_{1}^{\prime}}{2\left(1+\lambda_{0}\right)}+a_{1}^{\prime} a_{2}^{\prime}=56 \\
& \frac{-\left(a_{1}^{\prime} \lambda_{1}+a_{2}^{\prime} \lambda_{2}\right) a_{2}^{\prime}}{2\left(1+\lambda_{0}\right)}=12
\end{aligned}
$$

Simplifying (20) we get,

$$
\begin{aligned}
& \frac{\left(a_{1}^{\prime} \lambda_{1}+a_{2}^{\prime} \lambda_{2}\right)}{2\left(1+\lambda_{0}\right)}=\sqrt{\left(89-a_{1}^{\prime 2}-a_{2}^{\prime 2}\right)}= \\
& a_{2}^{\prime}-\frac{56}{a_{1}^{\prime}}=-\frac{12}{a_{2}^{\prime}}
\end{aligned}
$$

It is clear that (15) and (22) can be only satisfied only by $a_{1}^{\prime}=7$ and $a_{2}^{\prime}=2$. Substituting these values in (15) we get, $a_{0}^{\prime}=6$. So the polynomial with $a_{0}=6$, $a_{1}=7, a_{2}=2$, namely $A(z)=2 z^{2}+7 z+6$ is a stable polynomial. In this case, the given polynomial is found to be stable. For this polynomial $A(z)$, we find that the zeros are $|z|=3 / 2 \&|z|=2$.

As can be seen, this 1-D polynomial $A(z)$ is found to be stable as all its zeros lie outside the unit circle as said in theorem 3.1. Now when we check the stability of the remaining polynomials $A_{1}(z), A_{2}(z)$ and $A_{3}(z)$ that belong to the same family as that of $A(z)$, we find that at least one root of these polynomials lie inside the unit circle and hence unstable.

From this, it can be concluded that, out of these four polynomials that belong to a 'family' having the same ACF and hence magnitude response, only one polynomial is found to be stable for which the constant term is the highest. It is stressed that the above procedure to test the 1-D polynomial for stability is applicable only if the polynomial is nonlacunary, in the sense there are no missing terms between the highest degree and the constant term. When the given 
polynomial is lacunary, we have to make it nonlacunary and follow the same procedure.

\section{CONCLUSIONS}

In this paper, we proposed a new method for testing the stability and stabilizing 1-D recursive digital filters based on autocorrelation functions and Lagrange multipliers. It has been shown that maximizing the constant term of a denominator polynomial of a system transfer function will lead to a stable system whose magnitude response is similar to the magnitude response of the original system. The proposed method not only tests the stability of recursive digital filters, but also provides the stable version of the system. Like the LSI and DHT stabilization methods, this method also does not guarantee stability if the original denominator polynomial of the system transfer function has zeros on the unit circle. If the original polynomial has a zero on the unit circle, then all the polynomials in the family will have a zero on the unit circle and hence none of them are stable. The proposed method can be extended to testing the stability of 2-D recursive digital filters.

\section{REFERENCES}

1. A.Klouche-Djedid and S.S.Lawson, 2000. Design of doubly complementary filters with approximate linear phase, IEE Proc-Vision, Image, and Signal Processing, vol. 147, No. 2, pp. 103-108.

2. Jae S Lim, 1989. Two-dimensional signal and image processing, Prentice-Hall.

3. G.Proakis John and G.Manolakis Dimitris, 1999. Digital Signal Processing-Principles, Algorithms, and Applications, Prentice-Hall.

4. Dan E.Dudgeon and Russell M.Mersereau, 1984. Multidimensional Digital Signal Processing, Prentice-Hall.

5. E.A. Robinson, 1967. Statistical Communication and Detection, New York, Haftner, pp. 13-174.

6. Andreas Antoniou, 1993. Digital Filters-Analysis, Design and Applications, McGraw-Hill, Inc.
7. G.Schmeisser, D.Raghuramireddy, R.Unbehauen, 1986. An alternative proof for the stability of least squares inverse polynomials, IEEE Trans. Circuits and Systems, vol. 33, Issue. 8, pp. 822-824.

8. Niranjan Damera Venkata, Mahalakshmi Venkatraman, Hrishikesh and P.S.Reddy, 1999. Stabilization of 2-D recursive digital filters by the DHT method, IEEE Trans. Circuits and SystemsII: Analog and Digital Processing, vol. 40, No. 1, pp. 85-88.

9. B.D.O.Anderson and E.I.Jury, 1976. Proof of a Special Case of Shanks' Conjecture, IEEE Trans. Acoustics, Speech, and Signal Processing, pp. 574-575.

10. P.K.Rajan and H.C.Reddy, 1985. A note on LSI Polynomials and Comments on a Proof of a Modified Form of Shanks' Conjecture, IEEE Trans. Circuits and Systems, vol.CAS-32, No. 9, pp. 966-968.

11. P.S.Reddy, D.Raghu Rami Reddy, and M.N.S.Swamy, 1984. Proof of a Modified Form of Shanks' Conjecture on the Stability of 2-D Planar Least Square Inverse Polynomials and Its Implications, IEEE Trans. Circuits and Systems, vol.CAS-31, No.12.

12. John L Shanks, Sven Treitel, and James H Justice, 1972. Stability and Synthesis of two-dimensional recursive filters, IEEE Transactions on Audio and Electro Acoustics, vol.AU-20, No.2, pp. 115-128.

13. S.S.Rao, 1987. Optimization theory and applications, New Delhi, Wiley Eastern Limited.

14. N.Gangatharan and P.S.Reddy, 2003. The PLSI Method of Stabilizing Two-Dimensional Nonsymmetric Half Plane Recursive Digital Filters, EURASIP Journal on Applied Signal Processing, 9, pp. 914-921.

15. Kishan Shenoi, 1995. Digital Signal Processing in Telecommunications, Prentice Hall International Editions, USA. 